\title{
Colobronchial Fistula (CBF) Type 1 in a young female with advanced stage solid tumours with defective DNA mismatch repair (MSI-H)
}

\author{
Saleh S Alzahrani, Ehab Alsaggaf, Parvez AlamAldeein, Ashraf A Abdullah, Attaa Albaroudi and Sameh I Sersar* \\ King Abdullah Medical City, Makkah, Saudi Arabia
}

\begin{abstract}
We report a case of colobronchial fistula in a 43 years old female with history of advanced endometrial carcinoma involving ascending colon, spleen, pancreas and omentum. She underwent total hysterectomy, salpingoopharectomy, distal pancreatectomy, splenectomy right hemicolectomy and HIPEC with postoperative chemotherapy. later, she had colobronchial fistula that mandated laparotomy drainage of the collection, repair of transverse colon, excision of a fistulous track. Left thoracotomy and Closure of a diaphragmatic defect, clipping of the thoracic part of the fistulous track, wedge resection of the involved subsegment of the left lower lobe. Immediate resolution of the foul-smelling expectoration.
\end{abstract}

\section{Introduction}

Colobronchial fistula CBF is extremely rare. Very small number of cases is reported in the literature. A lot of causes are accused to be responsible for this pathology. The commonest cause is Crohn's disease, postoperative intraperitoneal adhesion and or diaphragmatic hernia. However, infections or abscesses, subphrenic collections, malignancy, radiotherapy and Hyper thermic intraperitoneal chemotherapy (HIPEC) have been reported as possible causes [1-5].

CBF can present with chronic expectoration with foul smelling sputum and rarely without foul smelling sputum, weight loss, fever, chest pain, dyspnoea and or haemoptysis. It is suspected when the Sputum culture grows intestinal flora positive, such as Escherichia coli.

$\mathrm{CBF}$ can be diagnosed by barium enema, CT scan with multiplanar reconstruction, flexible colonoscopy and may be post-mortem [1-3].

Surgical resection of the affected segment of the colon with or without lung resection is the main line of treatment. However, conservative treatment with antibiotics and total parenteral nutrition may be adopted $[4,5]$.

\section{Case Report}

A 44-year-old female presented, with endometrial cancer involving the surrounding structures and an unremarkable past medical history. Abdominal exploration was performed at our institution, revealing extensive peritoneal involvement of the pelvic organs, gastric nodule, pancreatic, splenic and ascending colon. Right hemicolectomy, total hysterectomy with salpingoopharectomy, distal pancreatectomy, splenectomy, omentectomy and HIPEC was performed. Pathological examination showed poorly differentiated carcinoma involving the left side of the uterine myometrium extending into the endometrium, with extensive lymphovascular invasion. The mesenteric lymph node showed metastatic poorly differentiated carcinoma. Splenectomy: showed poorly differentiated carcinoma involving the spleen, tail of pancreas, and omentum. With the tumour size: $10 \mathrm{~cm}$ in greatest dimension and the pancreatic surgical resection margin: uninvolved by carcinoma. Cecum, right colon and terminal ileum, right hemicolectomy uninvolved by carcinoma. With two metastatic poorly differentiated carcinoma deposits, the larger deposit size: $9 \mathrm{~cm}$ in greatest dimension with eleven lymph nodes, negative for metastatic carcinoma. Peritoneal deposit, biopsy: showed metastatic poorly differentiated carcinoma. Right ovary and tube were negative for malignancy. Gastric nodule, biopsy: showed spindle cell lesion involving a lymph node. The ileocecal lymph node biopsy showed reactive lymph node, negative for metastatic carcinoma. She had a smooth postoperative course and went home in a stable condition after 10 days. She tolerated the adjuvant chemotherapy well. Nine months postoperatively, she experienced refractory expectoration with foul odour sputum. Sputum culture grew E Coli She was started on broad spectrum antibiotics and CT chest and abdomen ordered which revealed left sub-phrenic collection and CBF (Figures 1a1c). Multidisciplinary meeting voted for redo laparotomy and left thoracotomy. The patient was consented. Redo laparotomy showed adhesions that were lysed, transverse colonic tear repaired, tumour in the pancreas which was resected, collection drained, Fistulous track dissected and clipped. Left thoracotomy was performed in the same operating session with closure of a diaphragmatic defect, clipping of the thoracic part of the fistulous track, wedge resection of the involved sub segment of the left lower lobe. (Figure 1d). Immediate resolution of the foul-smelling expectoration. Three months follow up was completely unremarkable apart from increased size of multiple right sided lung nodules mostly metastasis.

${ }^{*}$ Correspondence to: Sameh I Sersar, King Abdullah Medical City, Makkah, Saudi Arabia, E-mail: Ibrahim.S4@kamc.med.sa

Key words: colobronchoial fistula, cancer, surgery

Received: February 21, 2019; Accepted: March 07, 2019; Published: March 12 2019 


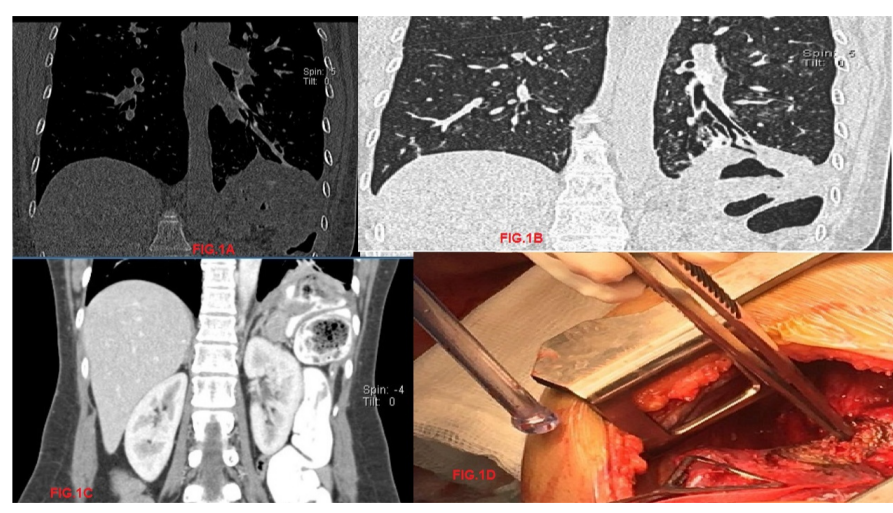

Figure 1: $(1 \mathrm{a}-1 \mathrm{c})$ CT Abdomen with oral and IV contrast showing the colobronchial fistulous track; 1d: Intraoperative Picture showing the diaphragmatic defect and the nontoothed forceps controlling the pulmonary end of the fistula

\section{Discussion}

The key of suspicion of colobronchial fistula was the refractory expectoration of foul odour with $\mathrm{E}$ coli in the sputum culture. The diagnosis was confirmed by CT chest and abdomen with oral and IV contrast. Patient was already on antibiotics before this presentation because of the unexplained? pneumonic infiltrates. The general condition of the patient was good, and her spirit was high. This may have helped her to overcome this pathology.

The splenic flexure was the affected area in our case. This is similar to the case reported by Karmy-Jones R, et.al.,1995 and Laterza B et.al., 2009 They explained the involvement of the splenic flexure by its cranial location, with direct contact to the left diaphragm with no protective role of the liver They added unnoticed injury of the left diaphragm during peritonectomy and or splenectomy with increased intraabdominal temperature and pressure during HIPEC [4,7].

Our patient and her family members are at increased risk for having an inherited colon cancer because she had loss of MLH1/PMS2 expression and defective DNA mismatch repair current data suggest that advanced stage solid tumours with defective DNA mismatch repair (MSI-H) are more likely to respond to treatment with immunotherapies such as anti-PD-1 therapies [8].

\section{References}

1. Kim SY, Park SH, Lee SS, Oh ST, Choi KD, et al (2008) Diagnosis of Colo-pulmonary fistula by virtual colonoscopy. Gastrointest Endosc 67: 769-771. [Crossref]

2. Zhao J, Ma N, Zhao Z, Lei J, Lu Q, et al. (2017) Colobronchial fistula: the pathogenesis, clinical presentations, diagnosis and treatment. J Thorac Dis 9: 187-193. [Crossref]

3. Kosugi K, Nishi T, Iijima T, Tanimoto T (2018) A case of colobronchial fistula causing foul-smelling sputum with a fecal odor. Intern Med 57: 761-762. [Crossref]

4. Laterza B, Baratti D, Cozzi G, Kusamura S, Oliva GD, et al. (2009) Colobronchial fistula: an unusual complication after peritonectomy and hyperthermic intra-peritoneal chemotherapy (HIPEC). In Vivo 23: 151-153. [Crossref]

5. Ashley S, Corlett SK, Windle R, Cookson JB (1988) Colobronchial fistula: a late complication of appendicitis. Thorax 43: 420-421. [Crossref]

6. Mercadal NR, Wiebke EA (2012) Recurrent pneumonia and colobronchial fistula from Crohn's disease: Infliximab alters and simplifies surgical management. Ann Gastroenterol 25: 361-364.

7. Karmy-Jones R, Chagpar A, Vallieres E, Hamilton S (1995) Colobronchial fistula due to Crohn's disease. Ann Thorac Surg 60: 446-448. [Crossref]

8. Le DT, Durham JM, Smith KN, Wang H, Bartlett BR et al. (2017) Mismatch repair deficiency predicts response of solid tumours to PD 1 blockade. Science 357: 409-413.

Copyright: (C2019 Alzahrani SS. This is an open-access article distributed under the terms of the Creative Commons Attribution License, which permits unrestricted use, distribution, and reproduction in any medium, provided the original author and source are credited. 